Bemerkugen.

1) Für Nr. 4 konnte kein Radiant abgeleitet werden. Schürholz angegebene Zeitdauer von $4^{5}$ richtig, so hatte das - 2) Nr. 24 war eine sehr helle, grün leuchtende Stern. Meteor in dem beobachteten Teile seiner Bahn eine Geschnuppe, welche für Utrecht $=$ Venus erschien, für Münster schwindigkeit von $22 \mathrm{~km}$ pro Sekunde. - 3) Es können sogar als Feuerkugel ihren Lauf beendete. Ist die von Herrn

tatsächlich nur Nr. 9 und Nr. 13 Leoniden genannt werden.

Schließlich möchten wir betonen, daß alle graphischen Ausgleichungen auf einer großen schwarzen Kugel ausgefübrt wurden.

Utrecht, 1902 Juli 9.

A. A. Nijland, F. v. d. Bilt.

\title{
Andamento delle latitudini eliografiche delle protuberanze solari, confrontato con quello delle macchie.
}

\author{
Di A. Riccò.
}

Nel $1893^{1}$ ) ho fatto vedere come dal 1880 in poi l' andamento delle latitudini eliografiche medie delle protuberanze era notevolmente parallelo a quello delle macchie solari: avendo ora continuato lo stesso confronto fino al 1901, ho trovato invece delle rilevanti discordanze, che credo opportuno di segnalare, poiche debbono avere un significato importante nella fisica solare.

Per questo studio ho confrontato le latitudini eliografiche medie annue delle protuberanze osservate e discusse in Catania dall' ing. Mascari ed in piccola parte da me, con le latitudini eliografiche medie annue delle macchie, misurate e calcolate nell' Osservatorio di Greenwich, sulle fotografie prese a Greenwich stesso, a Dehra Dûn (India), ed a Maurizio, le quali formano una serie completa ed affatto autentica, perchè desunta da fotografie ${ }^{2}$ ). La Tabella I e la Fig. I rappresentano questi dati.

Mass.

di frequenza

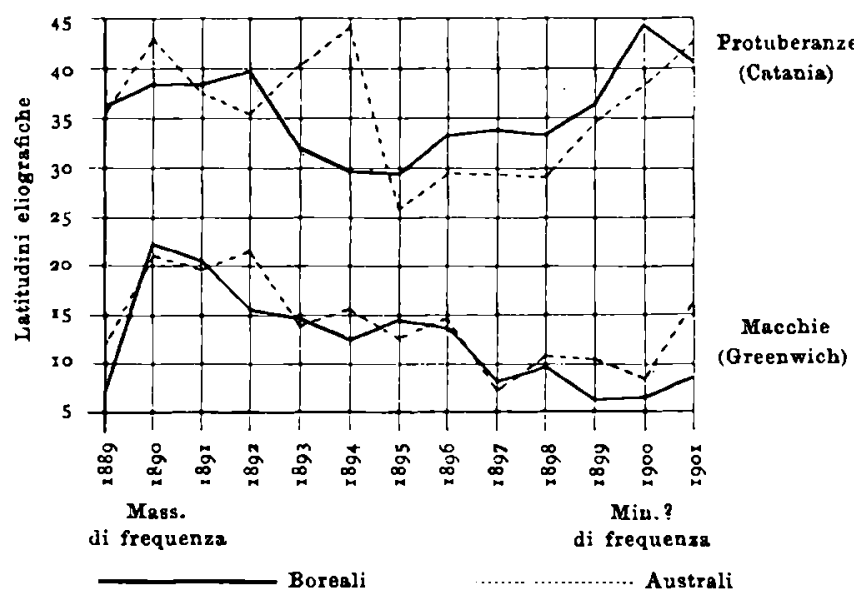

Fig. I.

Primieramente noterd che mentre nel periodo $1880-1892$ l' andamento delle latitudini eliografiche medie delle protuberanze e delle macchie boreali ed australi procedeva con notevole parallelismo: le latitudini essendo maggiori ora nell' uno ora nell' altro emisfero, e sol di poco; invece il secondo periodo presenta nel I893, e più ancora nel I894, una notevole anomalia, per cui le latitudini medie delle protuberanze sono molto maggiori nell' emisfero australe, che nel boreale, avendosi rispettivamente la media $+29: 7$ e $-44^{\circ} 3$. Questa singolarità devesi specialmente ad una straordinaria affluenza di protuberanze nel 1894 fra le altissime latitudini $-70^{\circ} \mathrm{e}-80^{\circ}$, ed anche oltre nelle regioni polari australi del sole: si aveva cosi nella distribuzione delle protuberanze per latitudine un massimo secondario rilevantissimo, oltre l' ordinario, pure importante alle latitudini basse col culmine fra $-20^{\circ}$ e $-30^{\circ}$. Dopo questo eccesso di protuberanze ad alte latitudini nell' emisfero australe, quasi per compenso in tutti gli anni seguenti fino al rgor la latitudine media delle protuberanze australi è sensibilmente più bassa di quella delle boreali.

Nulla di simile e avvenuto per le macchie, ove la curva della latitudini medie boreali si intreccia e si scosta poco da quella delle australi.

E da notare che nel 1893 , e più ancora nel 1894 , la frequenza complessiva delle protuberanze australi fu di molto superiore a quella delle boreali: però anche le macchie nei detti anni furono nell' emisfero australe assai più abbondanti che nel boreale.

Si deve anche rilevare che lo straordinario afflusso di protuberanze ad alta latitudine ha seguito di uno a due anni il massimo di frequenza delle macchie e delle protuberanze.

Considerando ora l' andamento complessivo delle lati. tudini medie delle protuberanze, e prescindendo dalla predetta anomalia del 1893 e 1894 , se lo si confronta con quello delle macchie, si puo ritenere che fin al 1895 si abbia un certo parallelismo, ma dopo, mentre le latitudini medie delle macchie continuano a scendere fino al 1899 , ed anche fino al r9oo (considerandole nel complesso dei due emisferi), per poi rialzarsi bruscamente nel rgor, le latitudini delle protuberanze dal $\mathbf{1} 895$ in poi salgono quasi continuamente e notevolmente fino al r900; cosicché il parallelismo nell' andamento dei due fenomeni non si ristabilisce in qualche modo che dal 1900 in poi.

1) Astronomische Nachrichten No. 3191, p. $3^{83}$.

2) Monthly Notices of R. A. S., March 1902, p. 378 . 
Si osserverà poi che in questo ciclo undecennale abbiamo il rialzo delle latitudini medie delle macchie nel 1901, ciod prossimamente all epoca del minimo attuale della loro frequenza, come nel ciclo precedente; invece il rialzo brusco delle latitudini delle protuberanze comincio nel 1899 cioè circa due anni prima del loro minimo, che come quello delle macchie, se non ebbe luogo nel rgor, avrà certamente luogo nel 1902 . Ció oltre alle predette discordanze indicherebbe una certa indipendenza fra $i$ due ordini di fenomeni, le macchie e le protuberanze solari.

\begin{tabular}{|c|c|c|c|c|}
\hline \multirow{3}{*}{ Anni } & \multicolumn{4}{|c|}{ Latitudini eliografiche medie } \\
\hline & \multicolumn{2}{|c|}{ Macchie solari } & \multicolumn{2}{|c|}{ Protuberanze } \\
\hline & Boreali & Australi & Boreali & Australi \\
\hline 1889 & $7: 3$ & $11: 9$ & $36: 4$ & $35^{\circ} \cdot 7$ \\
\hline 1890 & 22.2 & 21.7 & 38.5 & 42.9 \\
\hline $189 \mathrm{I}$ & 20.5 & 19.9 & 38.4 & $37 \cdot 9$ \\
\hline 1892 & 15.1 & 21.7 & 39.8 & $35 \cdot 5$ \\
\hline 1893 & 14.9 & $14 \cdot 3$ & 32.0 & 40.4 \\
\hline 1894 & $12 \cdot 3$ & 15.6 & 29.7 & $44 \cdot 3$ \\
\hline 1895 & $14 \cdot 3$ & $\mathbf{2} .5$ & 29.5 & 26.0 \\
\hline
\end{tabular}

Catania, I 902 Agosto.
Tabella I.

\begin{tabular}{|c|c|c|c|c|}
\hline \multirow{3}{*}{ Anni } & \multicolumn{4}{|c|}{ Latitudini eliografiche medie } \\
\hline & \multicolumn{2}{|c|}{ Macchie solari } & \multicolumn{2}{|c|}{ Protuberanze } \\
\hline & Boreali & Australi & Boreali & Australi \\
\hline 1896 & I $3: 6$ & $14: 8$ & $33^{\circ}: 4$ & $29: 5$ \\
\hline 1897 & 8.3 & $7 \cdot 7$ & 33.9 & 29.6 \\
\hline 1898 & 9.8 & 10.8 & 33.1 & 29.0 \\
\hline 1899 & 6.2 & 10.4 & 36.1 & 34.6 \\
\hline 1900 & 6.6 & $\mathbf{8 . 3}$ & 44.2 & $3^{8.9}$ \\
\hline 1901 & 8.6 & 16.3 & 40.6 & 42.6 \\
\hline
\end{tabular}

A. Riciò.

\section{Observations et éléments de l'étoile variable X Cygni (Ch. 7437).}

Par M. M. Luizet.

Cette étoile a été observée 272 fois, du 9 juillet 1898 au 15 janvier 1902 , par la méthode des degrés au moyen d'une jumelle de grossissement 5 environ. Les étoiles auxquelles elle a été comparée sont:

$$
\begin{array}{lr}
a_{1}=\mathrm{BD} .+35^{\circ} .4141 & \multicolumn{1}{c}{L} \\
a=\mathrm{BD} .+36.4105 & 10.7 \\
b=\mathrm{BD} .+34.4127 & 5.7 \\
d=\mathrm{BD} .+34.4111 & 2.3 \\
f=\mathrm{BD} .+34.4114 & 0.0
\end{array}
$$

Toutes ces observations ont été utilisées pour obtenir la courbe moyenne de lumière ci.dessous, où les temps $t$ sont comptés à partir du maximum, et les éclats de la variable donnés en degrés de mon échelle de lumière $L$.

\begin{tabular}{c|c}
\hline \multicolumn{1}{c|}{$t$} & \multicolumn{1}{c}{$L$} \\
\hline \hline 0.0 & 13.5 \\
+0.5 & 13.25 \\
+1.0 & 12.5 \\
+1.5 & 11.7 \\
+2.0 & 10.7 \\
+2.5 & 9.8 \\
+3.0 & 9.1 \\
+3.5 & 8.3 \\
+4.0 & 7.5 \\
+4.5 & 7.3 \\
+5.0 & 7.1 \\
+5.5 & 6.8
\end{tabular}

Cette courbe de lunière montre que:

10. L'éclat de l'étoile diminue pendant rọ̣z et aug. mente pendant 6.2.

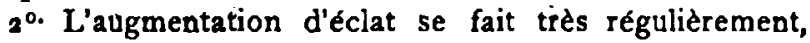
tandis que la diminution présente au contraire quelques irrégularités.

\begin{tabular}{c|l}
\hline$t$ & $L$ \\
\hline \hline+6.0 & 6.4 \\
+6.5 & 5.8 \\
+7.0 & 5.2 \\
+7.5 & 4.6 \\
+8.0 & 4.0 \\
+8.5 & 3.5 \\
+9.0 & 3.0 \\
+9.5 & 2.5 \\
+10.0 & 2.25 \\
+10.2 & 2.2 \\
+10.5 & 2.3 \\
+11.0 & 2.6
\end{tabular}

\begin{tabular}{c|r}
\hline$t$ & \multicolumn{1}{c|}{$L$} \\
\hline \hline+11.5 & 3.3 \\
+12.0 & 4.1 \\
+12.5 & 4.6 \\
+13.0 & 5.1 \\
+13.5 & 5.6 \\
+14.0 & 6.3 \\
+14.5 & 8.2 \\
+15.0 & 10.5 \\
+15.5 & 12.4 \\
+16.0 & 13.4 \\
+16.38 & 13.5 \\
\end{tabular}

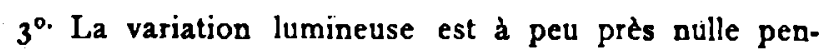
dant deux jours au voisinage du minimum.

A cause de cet aplatissement de la courbe, je me suis borné à la détermination des maxima; j'ai obtenu les 32 qui suivent: 